\title{
Incidence of Reactionary Haemorrhage Following Tonsillectomy Using Electrocautery Vs Ligation as Method of Haemostasis
}

\author{
${ }^{1}$ Dr. K. L. Shivkumar, ${ }^{2}$ Dr. Kamal Goyal, ${ }^{3}$ Dr. H. S. Satish, \\ ${ }^{1}$ Associate Professor, Department of E.N.T \\ ${ }^{2,3}$ Department of Otorhinolaryngology, Bangalore medical college and research institute, Bangalore - 560002,
} Karnataka, India.

Objectives: To compare electrocautery and ligation as method for haemostasis by comparing incidence of reactionary haemorrhage following tonsillectomy.

Study design: Prospective study.

Source of data: Data for this study was collected from the Department of Otorhinolaryngology, Venkateshwara ENT Institute \& Bowring Hospital, BMC \& RI, Bangalore

Sample size: The study included 100 patients, 50 each for electrocautery \& ligation.

Methods: 100 patients satisfying inclusion and exclusion criteria undergoing tonsillectomy; 50 each for ligation and electrocautery, who gave informed consent, were included in the study. Patients were followed for 24 hours. Method of haemostasis, incidence of haemorrhage with intervention documented.

Results: 2 patients in each group had reactionary haemorrhage.

Conclusion: Incidence of reactionary haemorrhage was same using electrocautery \& ligation as method of haemostasis.

Keywords: Tonsillectomy, Reactionary haemorrhage, Post operative pain

\section{Introduction}

Tonsillectomy is a surgical procedure in which tonsils are removed from the oropharynx. It is performed in cases of respiratory obstruction, peritonsillar abscess, sleep apnoea syndrome, chronic tonsillitis etc. The procedure is first mentioned in "Hindu medicine" about $1000 \mathrm{BC}$; roughly a millennium later the Roman Aristocrat Celsius (25 AD - 50 AD) described a procedure whereby using the finger (or a blunt hook if necessary), the tonsil was separated from the neighboring tissue prior to being cut out. Galen (121 - 200 AD) was the first to advocate the use of the surgical instrument known as the snare, a practice that was to become common until Aetius (490 AD) recommended partial removal of tonsil.

Today, the dissection method is still preferred for the removal of enlarged or recurrent infected tonsils in spite of various modern methods and surgical instruments. It is important to find the proper plane of dissection to avoid excessive bleeding. In spite of all the new surgical tools and techniques haemorrhage is still a significant complication during and after tonsillectomy and about 5\% patients may face this problem at any time from first 24 hours to 10 days after operation. Electro-cautery and suture ligation are the two common means for controlling haemorrhage during and after tonsillectomy with variable results.

\section{Patients \& Methods}

Source of data

Data for this study was collected from the Department of Otorhinolaryngology, Sri Venkateshwara ENT Institute \& Bowring Hospital, BMC \& RI, and Bangalore.

\section{Method of collection of data}

It was prospective study conducted from November 2011 to May 2013 in Bangalore medical college, Bangalore. The study included 100 patients. 50 each for electrocautery and ligation. Inclusion criteria included patients of all age group and both sexes selected to undergo tonsillectomy for chronic tonsillitis \& Hypertrophic-obstructive tonsils with symptoms such as snoring, apnoea or dysphagia. Exclusion criteria was patients undergoing tonsillectomy for glossopharyngeal neurectomy, Tonsillectomy for styloid process removal , Patients suffering from peritonsillar abscess two weeks pre-operatively \& Patients with chronic illnesses e.g. Diabetes, symptomatic heart disease, hemorrhagic diathesis, immunodeficiency and malignancy. 


\section{Ethical consideration}

The study protocol was approved by 'Ethics committee' of Bangalore medical college and research institute, Bangalore and an informed consent was obtained from all the participants.

\section{Methodology}

100 patients satisfying inclusion and exclusion criteria undergoing tonsillectomy; 50 each for ligation and electrocautery, who gave informed consent, were included in the study. Grading of the tonsils noted preoperatively. Method of haemostasis used \& duration of the operation was noted. Patients were followed up for 24 hours and incidences of haemorrhage with intervention, post operative pain at 6 hours, postoperative vomiting episodes were documented.

\section{Results}

A total of 100 patients were included in this study over a period of two years. In 50 patients electrocautery was used $\&$ in 50 ligation was used. Patients in whom cautery was used 31 ( $62 \%$ ) are male \& $19(38 \%)$ are female, 11 (22\%) belong to 5-10 year age group, $13(26 \%)$ belong to 11-15 year age group, 13 (26\%) belong to $16-21$ year age group , $3(6 \%)$ belong to $22-26$ year age group, $6(12 \%)$ belong to $27-31$ year of age group , $4(8 \%)$ belong to 32-35 year age group, 11(22\%) had Grade 2 tonsil, $30(60 \%)$ had Grade 3 tonsil, $9(18 \%)$ had Grade 4 tonsil; postoperatively after 6 hours, $36(72 \%)$ had pain score of $2,11(22 \%)$ had pain score of $3,3(6 \%)$ had pain score of 3 .

FACES pain scale is used in our study.

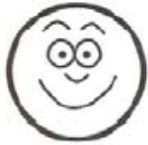
No hurt
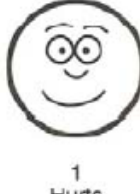

Hurts

little
bit

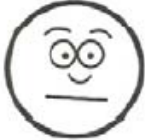

2

Hurts
little

little
more

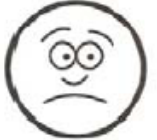

3

Hurts

oven

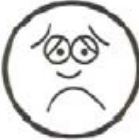

$4^{4}$

Hurts

Whole
lot

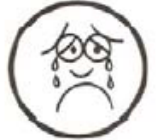

Hurts

worst

Patients in whom cautery was used as method of haemostasis $2(4 \%)$ patients had non blood stained minimal vomiting whereas $48(96 \%)$ had no complaint of vomiting.

Among the patients in whom ligation was used as method of haemostasis, $13(26 \%)$ are male \& 37 (74\%) are female, $13(26 \%)$ had Grade 2 tonsil, 31 (62\%) had Grade 3 tonsil, $6(12 \%)$ had Grade 4 tonsil, 2 (4\%) patients had reactionary haemorrhage, the same as that in patients, where electro-cautery was used i.e. $4 \%$ ( 2 patients out of 50 had reactionary haemorrhage).

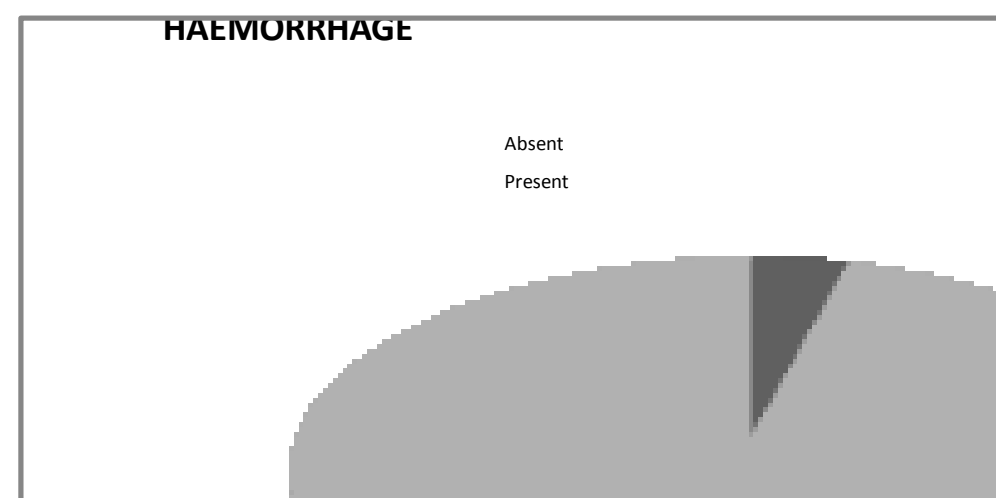

Among the 50 patients in whom ligation was used as method of haemostasis, in $3(6 \%)$ patients duration of operation was 20 minutes, in $16(32 \%)$ patients duration of operation was 25 minutes, in $17(34 \%)$ patients duration of operation was 30 minutes, in $11(22 \%)$ patients duration of operation was 35 minutes and in $3(6 \%)$ patients duration of operation was 40 minutes, postoperatively $20(40 \%)$ patients has a pain score of 2 , $26(52 \%)$ patients had pain score of 3 and $4(8 \%)$ patients had a pain score of 4 . 


\section{Discussion}

Tonsillectomy is one of the most commonly performed operations in paediatric population all over the world. Celsius and Paul described tonsillectomy in the literature in 1000 BC. The Greeks called the tonsils indurated and inflamed antiades. Different techniques and instruments have been used for removal of tonsil along with haemostasis but none of them were found satisfactory.

Modern methodologies like use of harmonic scalpel, bipolar scissor dissection, radio frequency excision with probes, microdebrider endoscopic tonsillectomy, laser tonsillectomy and the bipolar radio frequency ablation techniques have revolutionized the tonsillectomy surgery.

These new ways are considered to reduce the size of the tonsil, to decrease the time period, to minimize and prompt control of bleeding during surgery and to decline postoperative pain enabling the patient to resume his or her normal day-to-day activities.

In spite of all the new surgical tools and techniques haemorrhage is still a significant complication during and after tonsillectomy. Haemorrhage due to tonsillectomy has been classified according to the time i.e. primary occurring within 24 hours and secondary after 24 hours of surgery. The term reactionary haemorrhage is also used for intra-operative and primary haemorrhage.

* In our study we found that incidence of reactionary haemorrhage was same in both groups; as in each group, out of 50 patients $2(4 \%)$ patients had reactionary haemorrhage. In all the patients taken back to operation theatre following reactionary haemorrhage, we preferred ligation for haemostasis. In our study of hundred patients, we never encountered any case where secondary haemorrhage has occurred.

In study conducted by P.K.Moonka ${ }^{2}$, diathermy failed to achieve adequate haemostasis in 4 out of 188 cases \& Ligation was unsuccessful in 6 out of 188 cases. He concluded that bipolar diathermy is equally as effective as ligation in control of haemorrhage. In study conducted by Arif Raza Khan et $\mathrm{al}^{3}$ Primary haemorrhage was noticed in $4(6.66 \%)$ cases when haemostasis was achieved using bipolar diathermy and in $8(6.66 \%)$ cases when silk was used to control the bleeding during operation. The study conducted by Zeeshan Ayub ${ }^{4}$ showed monopolar diathermy as a better method for haemorrhage control as compared to silk ligatures. In study conducted by Karan Sharma ${ }^{5}$ he found average blood loss was remarkably reduced while using bipolar diathermy as compared to ligation.

* In our study, average duration taken for tonsillectomy operation where cautery was used as method of haemostasis was 27 minutes whereas duration taken for tonsillectomy operation using ligation as method of haemostasis is 29.5 minutes. According to statistical analysis this difference is significant $t$-value $1.45 \& \mathrm{p}$-value 0.015 . It reflects that time taken for tonsillectomy by using electrocautery as method of haemostasis is less saving theatre time \& less duration of general anaesthesia.

In study conducted by P.K. Moonka ${ }^{2}$, mean duration of operation in diathermy group was 15.5 minutes \& in ligation was 22.8 minutes. In study conducted by Arif Raza khan et al ${ }^{3}$ operation time was 15 minutes with bipolar diathermy as compared to 30 minutes on average for tonsillectomy by dissection snare method. Karan Sharma et $\mathrm{al}^{5}$ also found that tonsillectomy using bipolar diathermy was significantly quicker than that of tonsillectomy using ligation.

* In our study we used the FACES pain scale $0-5$ which is subjective scale where 0 is no hurt, 1 is hurt little bit , 2 is hurt little more, 3 is hurts even more, 4 is hurts whole lot and 5 is hurts worst. In the Electrocautery group average pain score is 2.34 whereas in ligation group average pain score is 2.68 . According to statistical analysis it is significant as t-value is $-2.26 \&$ p-value is 0.026 . So we found that patients in whom we used electrocautery had significantly less pain.

In study conducted by P.K.Moonka ${ }^{2}$ he found bipolar diathermy is not significantly more painful post operatively as ligation. In study conducted by Karan Sharma ${ }^{5}$ there was comparable postoperative pain in bipolar diathermy \& ligation methods.

* In our study, in Electrocautery group 2 patients (4\%) had postoperative vomiting, whereas in Ligation group 3 patients $(6 \%)$ patients had postoperative vomiting. According to statistical analysis this difference is insignificant as t-value is -1.70 and $\mathrm{p}$-value is 0.092 .

\section{Conclusion}

Incidence of reactionary haemorrhage using electrocautery or ligation as method of haemostasis during tonsillectomy is $4 \%$. Less duration is required to perform tonsillectomy using electrocautery as method of haemostasis; hence it saves theatre time \& prolonged exposure to general anaesthesia. Postoperative pain following electrocautery is significantly less as compared to ligation. We conclude that electrocautery is the preferred method of haemostasis over ligation as incidence of reactionary haemorrhage is the same while duration of operation is shorter \& postoperative pain is lesser. 
Incidence of Reactionary Haemorrhage Following Tonsillectomy Using Electrocautery Vs Ligation ...

Table 1: Relation and significant difference between cautery and ligation groups.

\begin{tabular}{|c|c|c|c|c|c|}
\hline SL & Variables & Coefficients & SD & t-Value & P-Value \\
\hline 01 & Age in Years & 45.01 & 8.23 & 5.47 & 0.011* \\
\hline 02 & Groups (Cautery and Ligation) & -3.04 & 1.58 & -1.93 & $0.047 *$ \\
\hline 03 & Grades & -4.33 & 1.23 & -3.52 & 0.001* \\
\hline 04 & Duration & 0.22 & 0.15 & 1.45 & $0.015^{*}$ \\
\hline 05 & Haemorrhage & -5.20 & 3.59 & -1.45 & $0.151^{\text {ns }}$ \\
\hline 06 & Pain Score & -2.83 & 1.25 & -2.26 & $0.026{ }^{*}$ \\
\hline 07 & Vomiting & -3.04 & 1.78 & -1.70 & $0.092^{\text {ns }}$ \\
\hline
\end{tabular}

\section{Bibliography}

[1]. McNeill R.A. (1960). A history of Tonsillectomy: Two Millennia of Trauma, Haemorrhage and controversy. Ulster Med. J. 29, 5963.

[2]. Moonka P.K. (2002) Ligation vs. Bipolar diathermy for haemostasis in tonsillectomy - A Comparative study. Indian J. Otolaryngol. Head Neck Surg. 54, 35-38.

[3]. Khan A.R., Khan A., Ali F. et al. (2007) Comparison between silk ligation and bipolar cautery in tonsillectomy. Gomal J. Med. Sci. 5,13-16.

[4]. Ayub Z., Ahmed B. (2009) Monopolar diathermy as compared to silk ligature for per operative haemorrhage control during tonsillectomy. Pakistan Armed Forces Medical Journal. 1-4

[5]. Sharma K., Kumar D. (2011) "Ligation versus Bipolar Diathermy for Haemostasis in Tonsillectomy": A Comparative Study; Indian J Otolaryngol Head Neck Surg. 63, 15-1 